\title{
Risk stratification in GIST: shape quantification with CT is a predictive factor
}

\author{
Sheng-cai Wei ${ }^{1} \cdot$ Liang $\mathrm{Xu}^{2} \cdot$ Wan-hu $\mathrm{Li}^{2} \mathrm{C}$ - Yun $\mathrm{Li}^{2} \cdot$ Shou-fang Guo ${ }^{2} \cdot$ Xiao-rong Sun ${ }^{1} \cdot$ Wen-wu $\mathrm{Li}^{2}$
}

Received: 27 August 2019 / Revised: 19 October 2019 / Accepted: 30 October 2019 / Published online: 3 January 2020

(C) The Author(s) 2020

\begin{abstract}
Background Tumor shape is strongly associated with some tumor's genomic subtypes and patient outcomes. Our purpose is to find the relationship between risk stratification and the shape of GISTs.

Methods A total of 101 patients with primary GISTs were confirmed by pathology and immunohistochemistry and underwent enhanced CT examination. All lesions' pathologic sizes were 1 to $10 \mathrm{~cm}$. Points A and B were the extremities of the longest diameter (LD) of the tumor and points $\mathrm{C}$ and $\mathrm{D}$ the extremities of the small axis, which was the longest diameter perpendicular to $\mathrm{AB}$. The four angles of the quadrangle $\mathrm{ABCD}$ were measured and each angle named by its summit $(\mathrm{A}, \mathrm{B}, \mathrm{C}, \mathrm{D})$. For regular lesions, we took angles $\mathrm{A}$ and $\mathrm{B}$ as big angle $(\mathrm{BiA})$ and small angle $(\mathrm{SmA})$. For irregular lesions, we compared $\mathrm{A} / \mathrm{B}$ ratio and $\mathrm{D} / \mathrm{C}$ ratio and selected the larger ratio for analysis. The chi-square test, $t$ test, ROC analysis, and hierarchical or binary logistic regression analysis were used to analyze the data.

Results The BiA/SmA ratio was an independent predictor for risk level of GISTs $(p=0.019)$. With threshold of $\mathrm{BiA}$ at $90.5^{\circ}$, $\mathrm{BiA} / \mathrm{SmA}$ ratio at 1.35 and $\mathrm{LD}$ at $6.15 \mathrm{~cm}$, the sensitivities for high-risk GISTs were $82.4 \%, 85.3 \%$, and $83.8 \%$, respectively; the specificities were $87.1 \%, 71 \%$, and $77.4 \%$, respectively; and the AUCs were $0.852,0.818$, and 0.844 , respectively. LD could not effectively distinguish between intermediate-risk and high-risk GISTs, but BiA could $(p<0.05)$. Shape and Ki-67 were independent predictors of the mitotic value ( $p=0.036$ and $p<0.001$, respectively), and the accuracy was $87.8 \%$.

Conclusions Quantifying tumor shape has better predictive efficacy than LD in predicting the risk level and mitotic value of GISTs, especially for high-risk grading and mitotic value $>$ 5/50HPF.

Key Points

- The BiA/SmA ratio was an independent predictor affecting the risk level of GISTs. LD could not effectively distinguish between intermediate-risk and high-risk GISTs, but BiA could.

- Shape and Ki-67 were independent predictors of the mitotic value.

- The method for quantifying the tumor shape has better predictive efficacy than LD in predicting the risk level and mitotic value of GISTS.
\end{abstract}

Keywords Gastrointestinal stromal tumor $\cdot$ Risk $\cdot$ Tomography $\cdot$ x-ray $\cdot$ Form

Xiao-rong Sun and Wen-wu Li contributed equally to this work.

Xiao-rong Sun

251400067@qq.com

Wen-wu Li

lwwzlm@outlook.com

1 Department of Nuclear Medicine, Shandong Cancer Hospital and Institute, Shandong First Medical University and Shandong Academy of Medical Sciences, No 440 Jiyan Road, Jinan 250117, Shandong Province, People's Republic of China

2 Department of Radiology, Shandong Cancer Hospital and Institute, Shandong First Medical University and Shandong Academy of Medical Sciences, No 440 Jiyan Road, Jinan 250117, Shandong Province, People's Republic of China

$\begin{array}{ll}\text { Abbreviations } \\ \text { Ap } & \text { Arterial phase } \\ \text { AUC } & \text { The area under the receiver } \\ & \text { operating characteristic curve } \\ \text { BiA } & \text { Big angle } \\ \text { CT } & \text { Computed tomography } \\ \text { GISTs } & \text { Gastrointestinal stromal tumors } \\ \text { FPF } & \text { High-power field } \\ \text { LD } & \text { Long diameter } \\ \text { ROC } & \text { Receiver operating characteristic } \\ \text { SD } & \text { Short diameter } \\ \text { SmA } & \text { Small angle } \\ \text { UE } & \text { Unenhanced }\end{array}$

Abbreviations

Arterial phase operating characteristic curve

CT Computed tomography

FPF High-power field

LD Long diameter

ROC Receiver operating characteristic

SmA Small angle

Unenhanced 


\section{Introduction}

Gastrointestinal stromal tumors (GISTs) are the most common mesenchymal tumors. Risk stratification of GISTs tries to evaluate the risk of poor outcome and to choose patients who may benefit from adjuvant therapy [1]. Although criteria may vary from country to country, the 2008 National Institute of Health (NIH) criteria are the most widely used. GISTs are classified into four categories (very low-, low-, intermediate-, and high-risk) according to tumor size, location, mitosis count, and tumor rupture [2]. Tumor size, location, and rupture can be evaluated by $\mathrm{CT}$, whereas mitosis count can only be obtained by pathological evaluation of the surgically removed specimen. Therefore, our primary purpose was to explore CT features that could predict the mitosis value before surgery. Tumor growth pattern or enlarged vessels feeding or draining the mass can help predict the risk [3]. CT features like location, contour, necrosis, enhancement pattern, and tumor calcification are associated with grades [4-6]; few studies report how tumor shape can be correlated with risk grading. In glioblastoma [7] or pancreatic neuroendocrine tumors [8], tumor shape is associated with the outcome. Therefore, we explored a novel method for quantifying the shape of GISTs using CT. In this method, we measured the LD and short diameter (SD) of the lesion and draw four angles $\mathrm{A}, \mathrm{B}, \mathrm{C}$, and $\mathrm{D}$. The $\mathrm{LD}$ corresponds to angle $\mathrm{A}$ and angle $\mathrm{B}(\mathrm{A} \geq \mathrm{B})$, and $\mathrm{SD}$ corresponds to angle $\mathrm{C}$ and angle $\mathrm{D}(\mathrm{D} \geq \mathrm{C})$. For regular lesions, we take angle $\mathrm{A}$ and angle $\mathrm{B}$ as $\mathrm{BiA}$ and $\mathrm{SmA}$. For irregular lesions, we compare $\mathrm{A} / \mathrm{B}$ ratio with $\mathrm{D} / \mathrm{C}$ ratio, select the larger ratio for analysis, and then investigate the relationship between risk stratification and the shape of GISTs.

\section{Materials and methods}

\section{Patients}

We searched the pathological database in our hospital from July 2014 to December 2018 using the search terms "GISTs." For study inclusion, the following criteria were used: (1) patients diagnosed with GIST by pathology and immunohistology after complete resection (laparoscopic, endo-luminal, or open surgery); (2) patients who had undergone unenhanced and tri-phasic $\mathrm{CT}$ before treatment and none of the lesions had tumor rupture at pathology; and (3) the lesion size was $\geq 1 \mathrm{~cm}$ and $<10 \mathrm{~cm}$ at pathology. The exclusion criteria were as follows: (1) patients with other history of another malignancy; (2) those who underwent any treatment before CT scan; (3) tumor rupture at pathology; and (4) the lesion size was $<1 \mathrm{~cm}$ and $\geq 10 \mathrm{~cm}$ at pathology. The study workflow diagram with respect to patient selection is shown in Fig. 1. Finally, we enrolled 101 patients (101 lesions in total):
49 males (mean age, 57.4 years \pm 11 [standard deviation]; range, 34-85 years) and 52 females (mean age, 57.4 years \pm 7.6 [standard deviation]; range, $45-80$ years). Among the 101 patients, only one had diffuse growth, and one case had two gastric lesions. In the case of two lesions, one of them grew irregularly and the other one grew regularly $(\mathrm{LD}=0.9 \mathrm{~cm})$, and we took the larger one for analysis. This study was approved by the institutional review board (IRB) at the Cancer Center, and informed consent was waived.

Among the 101 patients, 10 were asymptomatic, 38 had abdominal discomfort, 6 had loss of appetite or general fatigue, and 28 had bellyache or distension. In addition, 15 had melena, and 4 had obstructive symptoms such as vomiting. None of the patients underwent any type of therapy before the CT examination.

\section{Acquisition of $\mathrm{CT}$ images}

All 101 patients underwent tri-phasic abdominal or abdominopelvic CT scans. All CT scans were obtained using a high-speed 128 or 64 slice spiral CT scanner (Philips Brilliance iCT or Philips Brilliance $64 \mathrm{CT}$ ). The scanning parameters were as follows: $120 \mathrm{kV}, 250 \mathrm{mAs}, 32 \times 0.625$ collimation, 0.75 -s rotation time, 1.7 -s cycle time, and 5-mm increments. Each patient consumed $750-1000 \mathrm{~mL}$ of tap water or gastrointestinal oral contrast media (Aizhong Medical Imaging) approximately $60 \mathrm{~min}$ prior to, and an additional 500 -mL contrast immediately preceding $\mathrm{CT}$ imaging to maximize both bowel and gastric distension. The contrast medium used was Omnipaque $(300 \mathrm{mg} / \mathrm{L}$; General Electric) with 80 $100 \mathrm{~mL}$ injected via the median cubital vein at a rate of 2.7-3.0 $\mathrm{mL} / \mathrm{s}$. For the arterial phase (Ap), a delay time of $25-35 \mathrm{~s}$ was used. Venous phase $(\mathrm{Vp})$ and delayed phase $(\mathrm{Dp})$ scanning were performed $60-75 \mathrm{~s}$ and $90-120 \mathrm{~s}$ after contrast administration, respectively. The images were reconstructed with hybrid iterative reconstruction algorithm (iDose, level 3), standard kernel, contiguous 1-mm-thick slices. We reformatted contiguous 1-mm-thick coronal and sagittal images .

\section{Image analysis}

Two radiologists (LX and WHL) with 10-15 years of experience who were blinded to the pathology results independently reviewed the CT images of GIST on the Picture Archiving and Communication Systems Workstation (PACS). The two radiologists met later to reach a consensus on the results on which they had initially disagreed. The consensus results were used to analyze CT features of GISTs, and the results were from the independent reviews of each. The following CT findings were analyzed: location (stomach, small intestine, or others), contour (regular or irregular), growth pattern [3] (endoluminal, exophytic, or mixed), necrosis, enhancement pattern (homogenous or heterogeneous), and tumor calcification. 
Fig. 1 Flowchart showing the inclusion process. GISTs, gastrointestinal stromal tumors; LD, long diameter

\section{Consecutive patients with GISTs confirmed by pthology and immunohistology in our hospital's pathologic database from July 2014 to December $2018(\mathrm{~N}=419)$}

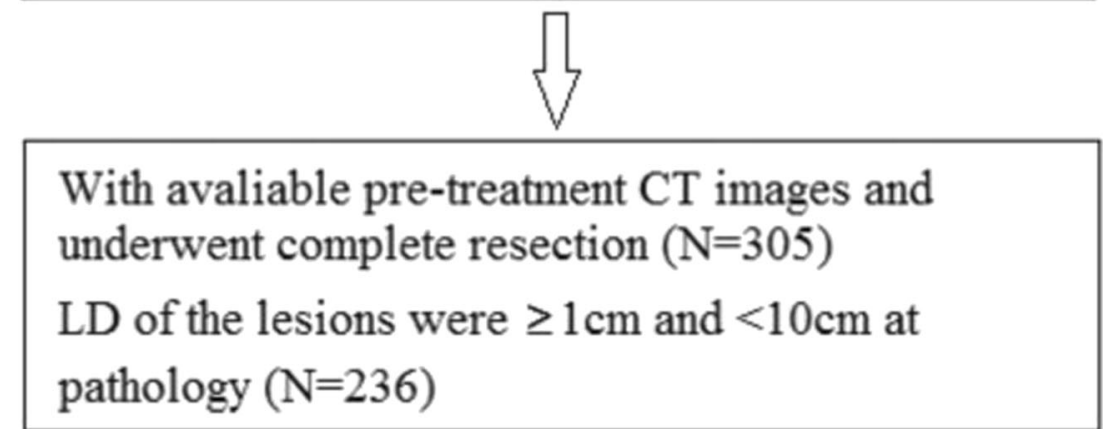

Another two radiologists (XRS and WWL) with 15-20 years of experience independently measured and recorded the unenhanced (UE) and tri-phasic CT attenuation coefficient (density) of the tumor in Hounsfield units (HU) by drawing a region of interest (ROI) of the solid components of lesions. The choice of ROI was determined as follows: the same level of ROI was used in each period; necrosis, calcification, hemorrhage, fibrosis, and obvious blood vessels were avoided; and the ROI was at least greater than $30 \mathrm{~mm}^{2}$. The CT attenuation coefficients (HU) of the lesion's same slices in the unenhanced, arterial, venous, and delayed phases were represented by UE, Ap, Vp, and Dp, respectively. Comparing the magnitude of the Ap, Vp, and Dp of the lesion, the phase of the maximum one was used as the enhanced peak period. The absolute enhancement CT attenuation coefficient of the Ap was represented by $\mathrm{ApU}$, and $\mathrm{ApU}$ was the subtraction value between the Ap and the UE; the absolute enhancement CT attenuation coefficient of $\mathrm{Vp}$ was represented by $\mathrm{VpU}$, and $\mathrm{VpU}$ was the subtraction value between the Vp and the UE; the absolute enhancement CT attenuation coefficient of Dp was represented by $\mathrm{DpU}$, and $\mathrm{DpU}$ was the subtraction value between the $\mathrm{Dp}$ and the UE.

The largest dimension of the lesion was selected when measuring the LD and SD of the lesion in the cross-sectional, coronal-reconstructed imaging, or sagittal reconstructed imaging (multiplanar reformation (MPR) imaging). Comparing the size of the cross-sectional, coronal, and sagittal planes of the tumor, the LD (line "AB") (Fig. 2) of the lesions was 
determined according to the Response Evaluation Criteria in Solid tumors [RECIST] [9], and then the maximum SD (line "CD") perpendicular to the LD was measured in this section. The four angles of the quadrangle $\mathrm{ABCD}$ were measured and each angle named by its summit (A, B, C, D). Angle measurements on the PACS system were very convenient, with three points determining an angle. The angle corresponding to the LD was angle $A$ and angle $B(A \geq B)$, and the angle corresponding to the $\mathrm{SD}$ was angle $\mathrm{C}$ and angle $\mathrm{D}(\mathrm{D} \geq \mathrm{C})$. Line
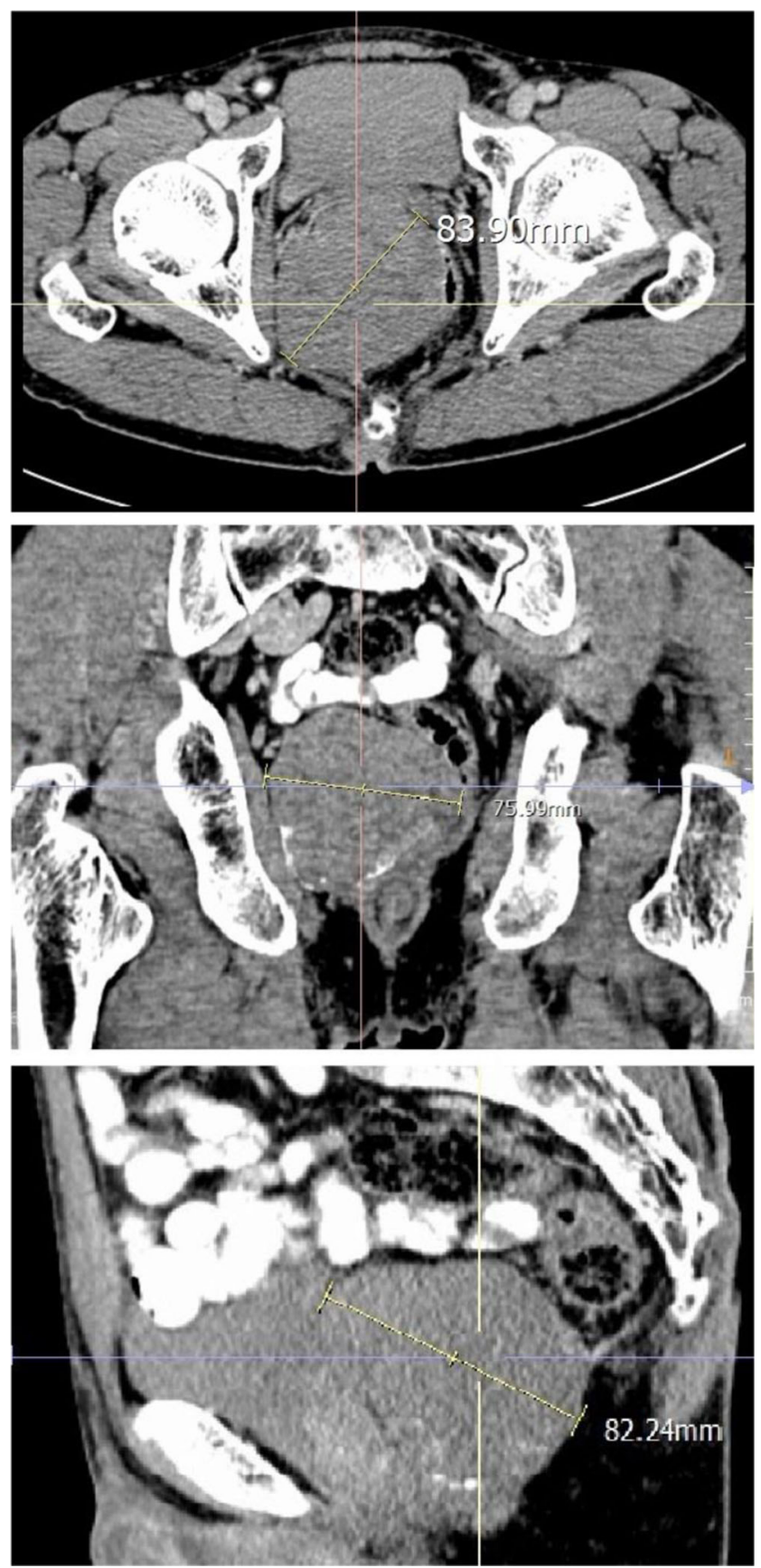

Fig. 2 Comparison of axial, coronal, and sagittal images of a pelvic tumor with sample measurements. The maximum value was selected to determine the long diameter (LD)
"AB" and line "CD" intersected at point "O." Point " $\mathrm{O}_{1}$ " and point " $\mathrm{O}_{2}$ " were the middle of line "AB" and line "CD." When the mass was regular, oval, or circular, we took the two opposite angles of the LD, angle A and angle B (Fig. $3 \mathrm{a}, \mathrm{b})$. For irregular lesions, if it was easy to discern the larger ratio of the angle $\mathrm{A} /$ angle $\mathrm{B}$ ratio to angle $\mathrm{D} /$ angle $\mathrm{C}$ ratio, we can directly measure the opposite angles corresponding to the larger ratio (Fig. 3c, d). If it was difficult to discern the two ratios, we would compare the angle $\mathrm{A} /$ angle $\mathrm{B}$ ratio with the angle $\mathrm{D} /$ angle $\mathrm{C}$ ratio and select the larger ratio for analysis (Fig. 3e, f). We used the pattern diagram to explain the irregular lesions in detail (Fig. 4). We took out the LD and SD of the lesion separately. In Fig. 4a, b, the LD and the SD intersected at point "O." When the LD crossed the midpoint of the SD or was within its vicinity, angle $\mathrm{D}$ was approximately equal to angle $C$. The closer point " $A$ " was to point "O," the larger the angle A was. The larger angle A/angle B ratio was, the mass would grow more prominently in the "OB" direction, and then the more irregular the whole lump was. The principles of Fig. $4 c, d$ are the same as Fig. $4 a, b$. If it was difficult to judge the size of the angle $\mathrm{A} /$ angle $\mathrm{B}$ ratio and the angle $\mathrm{D} /$ angle $C$ ratio visually (Fig. 4e), we measured each angle and calculated the angle ratio and then compared the two ratios and selected a larger ratio for analysis. Two of the authors (X and $\mathrm{W}$ ) independently measured the $\mathrm{LD}$ and $\mathrm{SD}, \mathrm{BiA}$, and the opposite $\mathrm{SmA}$ of each lesion, and the $\mathrm{LD} / \mathrm{SD}$ ratio and $\mathrm{BiA} /$ SmA ratio were calculated. For the length, angle, and CT attenuation coefficient to be measured, when the difference between the two radiologists was within $0.1 \mathrm{~cm}, 1^{\prime}$ and 1 $\mathrm{HU}$, respectively, the average was taken. If this range was exceeded, the two authors would negotiate together to find out the cause of the measurement error and re-measured to determine the final result. The values of measured LD, SD, $\mathrm{LD} / \mathrm{SD}$ ratios; $\mathrm{BiA}, \mathrm{SmA}$, and $\mathrm{BiA} / \mathrm{SmA}$ ratios; and $\mathrm{UE}, \mathrm{ApU}$, $\mathrm{VpU}$, and $\mathrm{DpU}$ were used in the analysis.

\section{Statistical analysis}

In our study, we divided the mitotic values into $\leq 5$ and $>5$ per 50 HPF (high-power field) two levels, dividing the risk classification into very low- or low-, intermediate-, and high-risk three levels. To analyze whether there were significant differences between risk grading and $\mathrm{CT}$ features, $\mathrm{BiA}, \mathrm{BiA} / \mathrm{SmA}$ ratio, $\mathrm{LD}$, or $\mathrm{LD} / \mathrm{SD}$ ratio, we used the chi-square test for the categorical variables and the $t$ test or nonparametric test for the continuous variables. A $p$ value of less than 0.05 was considered to indicate a significant difference. Receiver operating characteristic (ROC) analysis was performed to determine the optimal cutoff value of the $\mathrm{BiA}, \mathrm{BiA} / \mathrm{SmA}$ ratio, $\mathrm{LD}$, and $\mathrm{LD} / \mathrm{SD}$ ratio differentiating the high-risk GISTs and intermediate-risk grade or differentiating the mitosis values $\leq 5$ and $>5 / 50 \mathrm{HPF}$. The correct index, also known as the Youden index, was the sum of sensitivity and specificity 

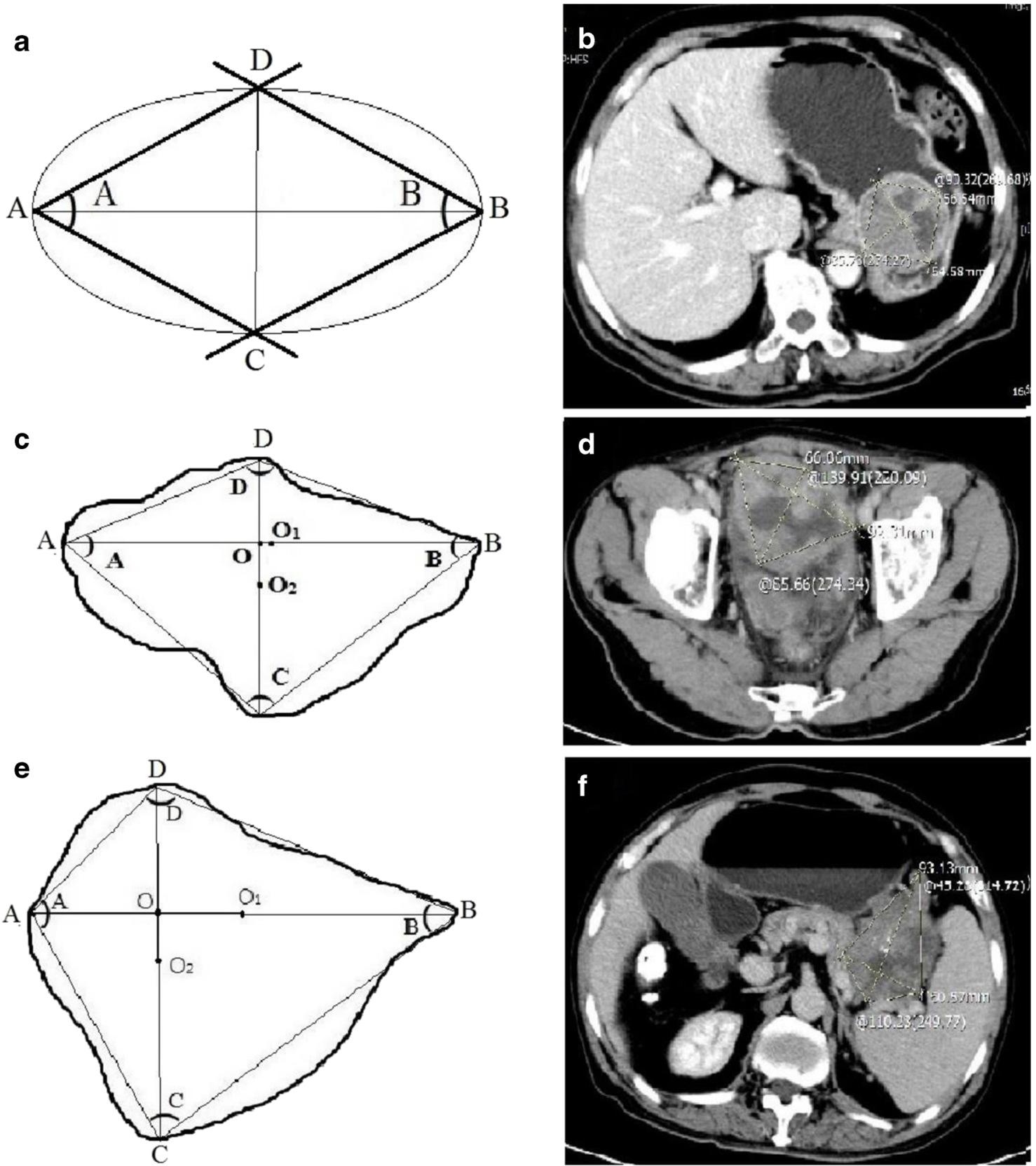

Fig. 3 Two-dimensional schematic diagram and actual tumor measurement picture of three types of GISTs with different shapes. a, b For the regular oval or circular lesions, we took angle A $\left(90.32^{\prime}\right)$ and angle $\mathrm{B}\left(85.73^{\prime}\right)$ as the big angle (BiA) and the small angle (SmA). Then, we obtained the $\mathrm{BiA} / \mathrm{SmA}$ ratio $\left(90.32^{\prime} / 85.73^{\prime}\right)$, which was approximately 1.05 . Then, the tumor was classified as intermediate risk

or below, and it was pathologically classified as an intermediate-risk GIST. $\mathbf{c}-\mathbf{f})$ When the lesions were irregular, the $\mathrm{BiA} / \mathrm{SmA}$ ratios were angle A/angle B $\left(139.91^{\prime} / 65.66^{\prime}=2.13\right)$ and angle D/angle C $\left(110.23^{\prime}\right.$ $\left.45.10^{\prime}=2.44\right)$, respectively. Both tumors were classified as high-risk GISTs, which was consistent with the pathological risk classification

minus 1. In the Excel table, the results of the subtraction were sorted to obtain the maximum value of the correct index, which was the optimal cutoff. Then, the maximum sum of specificity and sensitivity was obtained. Hierarchical logistic regression analysis was used to identify independent influencing factors affecting the risk grading of GISTs, using binary logistic regression analysis to identify independent influencing factors affecting the mitosis values. In addition, some data

groupings would be reasonably merged in the analysis because of the need.

\section{Results}

The relationship between qualitative and quantitative $\mathrm{CT}$ findings was presented in Tables 1 and 2. The ROC curves for LD, 
a

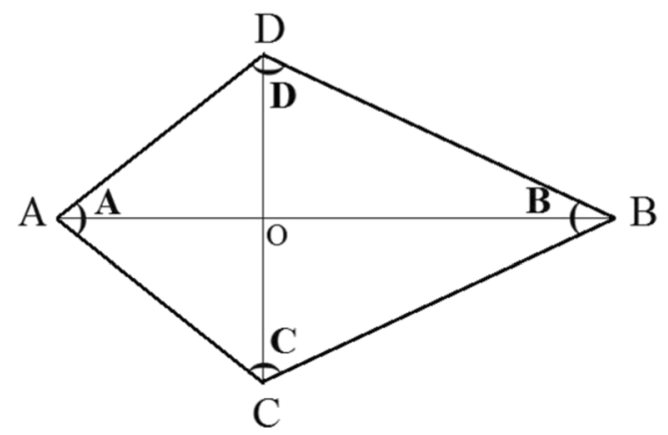

C

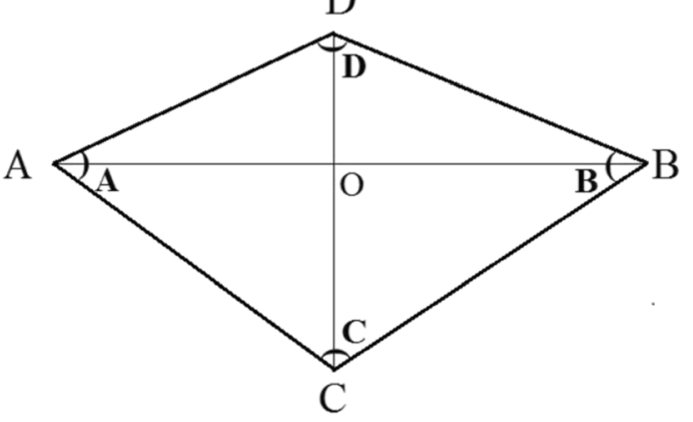

e

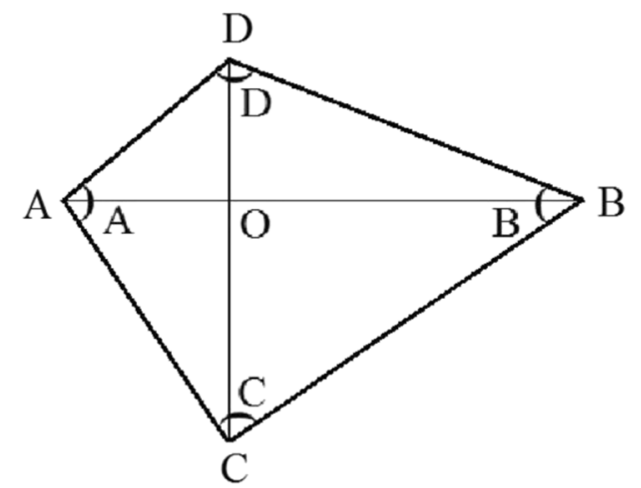

Fig. 4 For understanding, we isolate the $\mathrm{LD}$ and $\mathrm{SD}$ of the irregular lesion for analysis. a, b Angle A/angle B ratio is larger than angle D/ angle $C$ ratio visually, and angle $\mathrm{D} /$ angle $C$ ratio is close to 1 . When point " $\mathrm{A}$ " is closer to the point "O", the value of angle A/angle B ratio will be larger, and the lesion grows toward the "OB" direction as a whole, and the

$\mathrm{BiA}$, and $\mathrm{BiA} / \mathrm{SmA}$ were shown in Fig. 5. It included the best cutoff value for distinguishing high-risk and other risks of GISTs and their sensitivity, specificity, and the area under curve (AUC) values. In addition, LD could not effectively distinguish between intermediate-risk and high-risk GISTs $(p=0.057)$, but BiA could distinguish them $(p<0.05)$. The ROC curve for BiA to distinguish high-risk from intermediaterisk GISTs was shown in Fig. 6a. For GISTs, the LD, location, and $\mathrm{BiA} / \mathrm{SmA}$ ratio were the independent influencing factors affecting the risk classification by hierarchical logistic b

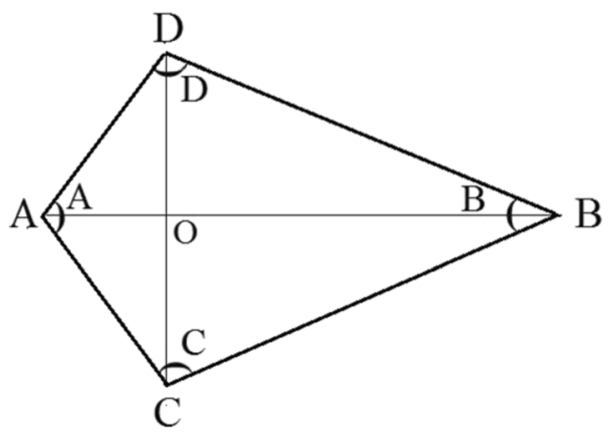

d

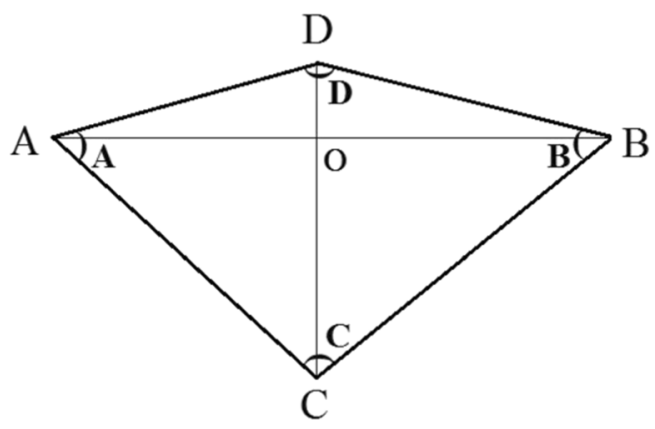

lesion is more irregular as a whole. According to the results of our analysis, the risk stratification of the lesion will be higher. The explanations of (c) and (d) are the same as (a) and (b). e When it is difficult to judge the values of angle $\mathrm{A}$ /angle $\mathrm{B}$ ratio and angle $\mathrm{D} /$ angle $\mathrm{C}$ ratio, we will compare the two ratios and select the larger ratio for analysis

regression analysis, and the $p$ values were $0.005(95 \% \mathrm{CI}=$ 1.381 7.737), $0.006(95 \% \mathrm{CI}=-51.103 \sim 9.156)$, and 0.019 $(95 \% \mathrm{CI}=3.349 \sim 37.079)$, respectively. In the bivariate correlation analysis, the Spearman correlation coefficients of LD with $\mathrm{BiA}$ and $\mathrm{BiA} / \mathrm{SmA}$ ratio were 0.586 and 0.622 , respectively $(p<0.001$, respectively), indicating that $\mathrm{LD}$ was moderately positively correlated with $\mathrm{BiA}$ and $\mathrm{BiA} / \mathrm{SmA}$ ratio. Although the $\mathrm{BiA}$ and $\mathrm{BiA} / \mathrm{SmA}$ ratio had some correlation with $\mathrm{LD}$, there were still many lesions with small size but very irregular, and some lesions were large but very regular. 
Table 1 Relationship between risk grading and sex, qualitative CT findings or Ki-67 index

\begin{tabular}{|c|c|c|c|c|c|}
\hline & \multicolumn{3}{|l|}{ Risk grading } & \multirow[t]{2}{*}{$X^{2}$ value } & \multirow[t]{2}{*}{$p$ value } \\
\hline & $\mathrm{VL}$ and $\mathrm{L}$ & Intermediate & High & & \\
\hline Sex & & & & 1.816 & 0.403 \\
\hline Men & $19(46.3 \%)$ & $11(40.7 \%)$ & $19(57.6 \%)$ & & \\
\hline Women & $22(53.7 \%)$ & $16(59.3 \%)$ & $14(42.4 \%)$ & & \\
\hline Growth $^{\mathrm{b}}$ pattern & & & & $20.318^{\mathrm{a}}$ & $<0.001$ \\
\hline Endoluminal & $21(52.5 \%)$ & $10(37 \%)$ & $2(6.3 \%)$ & & \\
\hline Exophytic & $12(30 \%)$ & $13(48.1 \%)$ & $28(87.5 \%)$ & & \\
\hline Mixed & $7(17.5 \%)$ & $4(14.8 \%)$ & $2(6.3 \%)$ & & \\
\hline Calcification & & & & 6.606 & 0.037 \\
\hline Absent & $38(92.7 \%)$ & $21(77.8 \%)$ & $23(69.7 \%)$ & & \\
\hline Present & $3(7.3 \%)$ & $6(22.2 \%)$ & $10(30.3 \%)$ & & \\
\hline Necrosis & & & & 19.444 & $<0.001$ \\
\hline Absent & $35(85.4 \%)$ & $12(44.4 \%)$ & $13(39.4 \%)$ & & \\
\hline Present & $6(14.6 \%)$ & $15(55.6 \%)$ & $20(60.6 \%)$ & & \\
\hline Shape $^{c}$ & & & & 38.208 & $<0.001$ \\
\hline Regular & $37(90.2 \%)$ & $13(48.1 \%)$ & $6(18.8 \%)$ & & \\
\hline Irregular & $4(9.8 \%)$ & $14(51.9 \%)$ & $26(81.2 \%)$ & & \\
\hline Enhancement pattern & & & & 16.191 & $<0.001$ \\
\hline Heterogeneous & $14(34.1 \%)$ & $18(66.7 \%)$ & $26(78.8 \%)$ & & \\
\hline Homogeneous & $27(65.9 \%)$ & $9(33.3 \%)$ & $7(21.2 \%)$ & & \\
\hline Enhanced peak period $^{\mathrm{d}}$ & & & & $33.726^{\mathrm{a}}$ & $<0.001$ \\
\hline Arterial phase & $2(4.9 \%)$ & $1(3.7 \%)$ & $17(53.1 \%)$ & & \\
\hline Venous phase & $7(17.1 \%)$ & $6(22.2 \%)$ & $8(25 \%)$ & & \\
\hline Delay period & $32(78 \%)$ & $20(25 \%)$ & $7(21.9 \%)$ & & \\
\hline $\mathrm{Ki}-67$ & & & & $28.238^{\mathrm{a}}$ & $<0.001$ \\
\hline$\leq 5 \%$ & $40(97.6 \%)$ & $25(92.6 \%)$ & $17(51.5 \%)$ & & \\
\hline$>5 \%$ & $1(2.4 \%)$ & $2(8.4 \%)$ & $16(48.5 \%)$ & & \\
\hline
\end{tabular}

$V L$, very low-risk; $L$, low-risk

${ }^{a}$ Kruskal-Wallis test

${ }^{\mathrm{b}}$ One case was diffuse growth and the other was multiple gastric GISTs

${ }^{\mathrm{c}}$ In a case of multiple gastric GISTs, one grew regularly and one grew irregularly

${ }^{\mathrm{d}}$ One lesion had no delay period

Five CT features or pathological results were significantly suggestive of mitotic value $>5$ per $50 \mathrm{HPF}$ rather than $<5$ per $50 \mathrm{HPF}$ : exophytic growth pattern $(p=0.015)$, irregular shape $(p=0.001)$, bigger BiA $(p=0.007), \mathrm{ApU}(p=0.008)$, and Ki67 index (> 5\%) $(p<0.001)$, However, shape and Ki-67 index were independent factors of the mitotic value by binary logistic regression analysis, and the $p$ values were $0.036(95 \% \mathrm{CI}=$ 1.117 26.123) and less than $0.001(95 \% \mathrm{CI}=2.857 \sim 40.654)$ respectively. The regular shape or lower Ki-67 index indicated lower mitotic counts, and the accuracy of prediction was $87.8 \%$. When we set the cutoff value for the BiA at $89.5^{\circ}$, the sensitivity and specificity for mitotic value $>5 / 50 \mathrm{HPF}$ were $70.4 \%$ and $70.6 \%$, respectively, and the AUC was 0.71 (Fig. 6b)

\section{Discussion}

The method for quantifying tumor shape can predict the risk level and mitotic value of GISTs, especially for high-risk grading and mitotic value $>5 / 50 \mathrm{HPF}$. BiA has better predictive efficacy than LD in distinguishing between intermediate-risk and high-risk GISTs or high-risk and other risks GISTs.

Enhancement CT is the standard method for GIST imaging [10] and plays an important role in the preoperative evaluation of GISTs [6, 11-13]. Some measuring tools of PACS [14, 15] could facilitate the diagnosis and risk assessment of GISTs. Computer-extracted shape cues could be used to distinguish radiographically similar pathologies of adenocarcinomas from granulomas on the lung [16]. Zanoni et al [17] found that shape may be a variant source of the tumor. For a solid tumor 
Table 2 Relationship between risk grading and age or quantitative CT findings

\begin{tabular}{lcccr}
\hline & \multicolumn{2}{c}{ Risk grading } & \multirow{2}{*}{$p$ value } \\
\cline { 2 - 4 } & VL and L & Intermediate & High & \\
\hline Age (year) & $57.6 \pm 10.9$ & $57.9 \pm 7.4$ & $55.4 \pm 8.5$ & $0.198^{\mathrm{a}}$ \\
LD (cm) & $3.0 \pm 1.3$ & $5.9 \pm 1.9$ & $6.9 \pm 1.8$ & $<0.001^{\mathrm{b}}$ \\
LD/SD & $1.25 \pm 0.15$ & $1.28 \pm 0.17$ & $1.32 \pm 0.26$ & $0.786^{\mathrm{a}}$ \\
BiA (') & $78 \pm 7$ & $95 \pm 16$ & $108 \pm 18$ & $<0.001^{\mathrm{a}}$ \\
BiA/SmA & $1.07 \pm 0.07$ & $1.32 \pm 0.31$ & $1.62 \pm 0.52$ & $<0.001^{\mathrm{a}}$ \\
plain scan (HU) & $36 \pm 6$ & $32 \pm 7$ & $36 \pm 6$ & $0.056^{\mathrm{a}}$ \\
Ap (HU) & $16 \pm 12$ & $15 \pm 12$ & $36 \pm 24$ & $<0.001^{\mathrm{a}}$ \\
Vp (HU) & $27 \pm 16$ & $26 \pm 15$ & $35 \pm 16$ & $0.056^{\mathrm{a}}$ \\
Dp (HU) & $28 \pm 12$ & $32 \pm 16$ & $32 \pm 13$ & $0.228^{\mathrm{a}}$ \\
\hline
\end{tabular}

${ }^{\mathrm{a}}$ Kruskal-Wallis test

${ }^{\mathrm{b}}$ One-way ANOVA

to grow in a confined space defined by the surrounding tissue, it must overcome the resulting compressive forces. Cheng et al [18] found a strong correlation between the peri-spheroid solid stress distribution and spheroid shape, a result of the suppression of cell proliferation and induction of apoptotic cell death in regions of high mechanical stress. In addition, Mazurowski et al [19] reported that shape features were strongly associated with genomic subtypes and patient outcomes in lower-grade glioma, and Okabe et al [8] reported that irregular tumor shape on preoperative computed tomography was a negative prognostic factor after curative surgery for pancreatic neuroendocrine tumors. Therefore, we believe that there is a significant correlation between the shape of GIST and risk level. Most studies divided tumors into regular and irregular shapes [3, 5], but in our study, we quantified the tumor shape, transforming qualitative analysis into quantitative analysis on PACS by CT threedimensional reconstruction imaging, which, to our knowledge, has not been previously reported. According to the improved classification of GISTs proposed by the NIH in 2008, lesion size, location, and rupture were used as independent factors in predicting the risk grading in the preoperative $\mathrm{CT}$ findings [2]. In this study, the $\mathrm{BiA} / \mathrm{SmA}$ ratio is verified as an independent influencing factor affecting the risk classification. We also find that the $\mathrm{BiA}$ or $\mathrm{BiA} / \mathrm{SmA}$ ratio has better effect on predicting grading of GISTs less than $10 \mathrm{~cm}$ than LD, especially in distinguishing between intermediate-risk and high-risk GISTs or high-risk and other risk GISTs. In addition, quantifying the shape to study the risk grading of GISTs is the highlight of this research.

Tumor mitotic figure is one of the independent prognostic factors $[2,20,21]$. $\mathrm{Ki}-67$ is a proliferation marker for tumors and has been used for tumor staging, poorly differentiated malignancies [22]. Li et al [23] reported that the $\mathrm{Ki}-67$ index was positively correlated with mitotic counts. Meanwhile, Kemmerling et al [24] reported that $\mathrm{Ki}-67$ could accurately predict mitotic
Fig. 5 Receiver operating characteristic curve of the LD, $\mathrm{BiA}$, and $\mathrm{BiA} / \mathrm{SmA}$ ratio for identifying high-risk GISTs. When we set the cutoff value for $\mathrm{BiA}$ as $90.5^{\circ}, \mathrm{BiA} / \mathrm{SmA}$ ratio as 1.35 , and long diameter (LD) as $6.15 \mathrm{~cm}$, the sensitivity values for high-risk GISTs were $82.4 \%$, $85.3 \%$, and $83.8 \%$, respectively; the specificity values were $87.1 \%$, $71 \%$, and $77.4 \%$, respectively; and the AUC values were 0.852 , 0.818 , and 0.844 , respectively. AUC, area under the curve; Sens, sensitivity; Spec, specificity

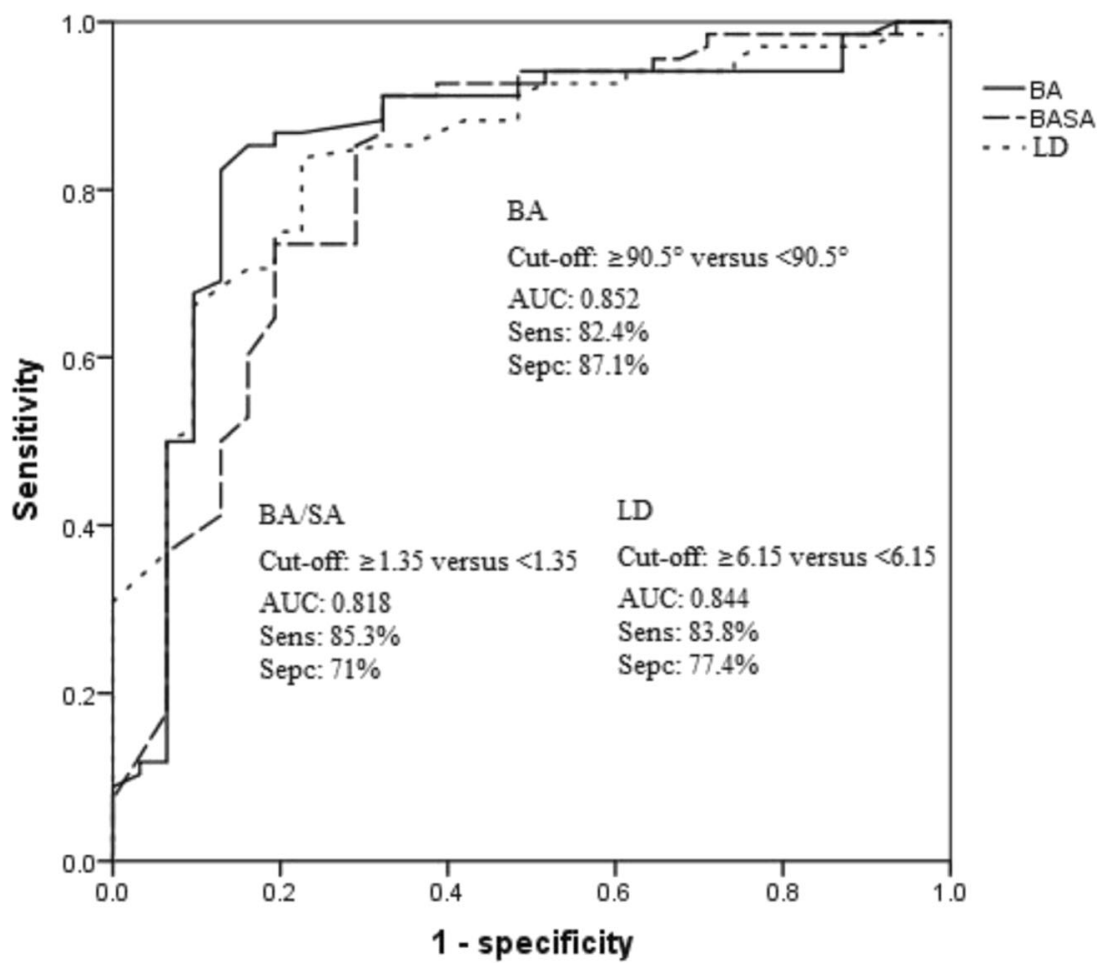


a

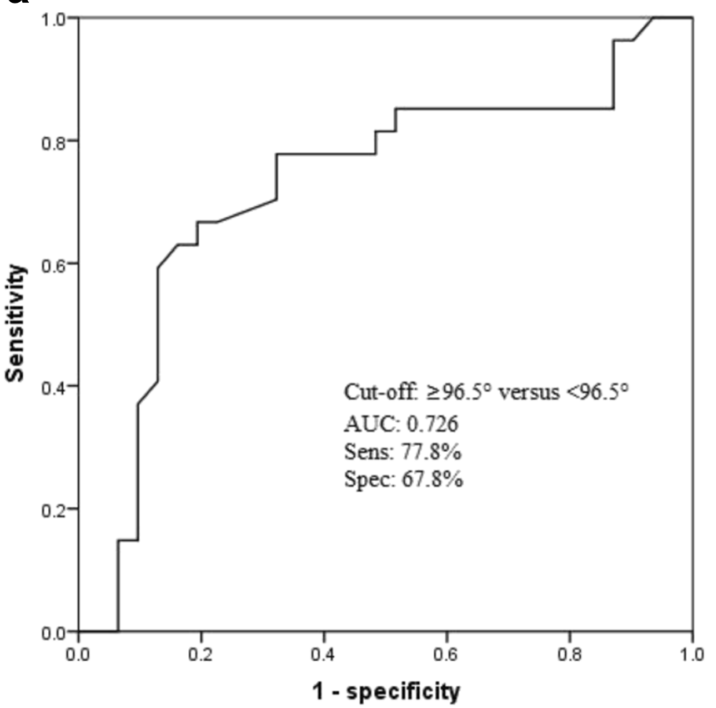

Fig. 6 a When we set the cutoff value for BiA at $96.5^{\circ}$, the sensitivity and specificity for distinguishing high-risk from intermediate-risk GISTs were $77.8 \%$ and $67.8 \%$, respectively, and the AUC value was 0.726 . b When we set the cutoff value for $\mathrm{BiA}$ at $89.5^{\circ}$, the sensitivity and specificity for

counts. Our research was consistent with them. In our study, GISTs with mitotic value > 5/50 HPF more commonly had an exophytic growth pattern, irregular shape, bigger $\mathrm{BiA}$ and $\mathrm{Ap}$, and $\mathrm{Ki} 67$ index (> 5\%), but only shape and Ki-67 index were independent factors of mitotic value by hierarchical logistic regression analyses. This finding was different from that in a previous study [25], in which size was the only significant predictor of high mitotic counts. However, there was no significant difference between the mitotic value and different sizes ( $p=0.075)$ in our research, and our research was very similar to that of Chen et al [26], in which there was no difference between mitotic counts and size, while the difference between mitotic counts and shape or growth patterns was statistically significant. In addition, some studies evaluated tumor response to treatment with CT attenuation coefficient on enhanced CT [27, 28], but they did not study the relationship between triphasic $\mathrm{CT}$ attenuation coefficients and mitotic value. In our study, the absolute CT attenuation coefficient of the arterial phase $(\mathrm{ApU})$ could predict the mitotic value to a certain extent, and the lesion with a higher $\mathrm{ApU}$ value was more likely to have a higher mitotic value. The higher the ApU value was, the more abundant the blood supply of the tumor was. Therefore, it is suspected that the mitotic value may be related to the blood supply mode of GISTs, but the relationship between mitotic value and Ap still needs further research.

There were several limitations in our present study. First, the evaluation of cases was retrospective, making selection bias unavoidable. Second, all patients did not

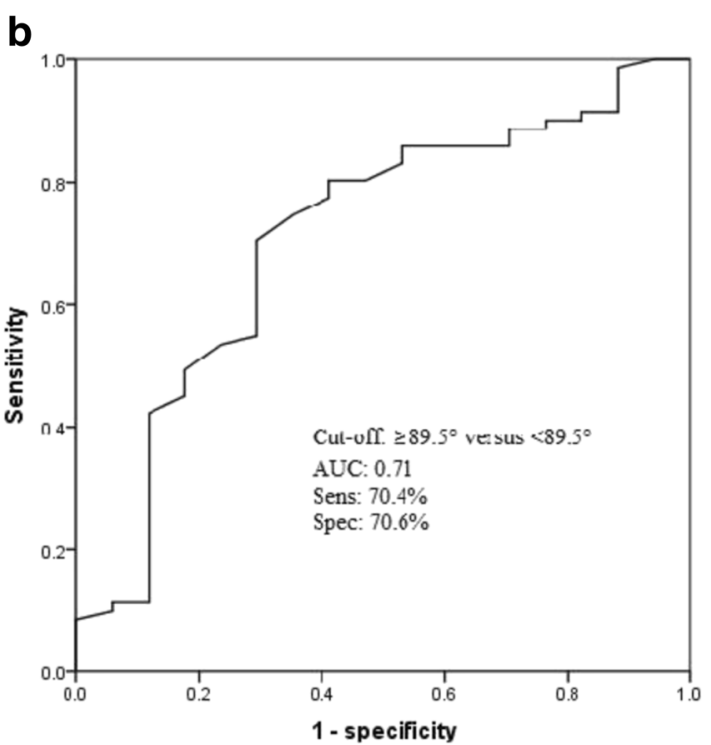

mitotic counts $>5 / 50 \mathrm{HPF}$ were $70.4 \%$ and $70.6 \%$, respectively, and the AUC was 0.71. AUC, area under the curve; Sens, sensitivity; Spec specificity

undergo a uniform pre-examination of gastrointestinal preparation, as some patients used warm water, and some used oral gastrointestinal contrast agents, which would have some impacts on the results. Third, the number of cases in the retrospective study remained small, and some of the data subgroups had to be combined to reach statistically relevant class size.

In conclusion, the method for quantifying the tumor shape can predict the risk level and mitotic value of GISTs, especially for high-risk grading and mitotic value $>5 / 50 \mathrm{HPF}$. BiA has better predictive efficacy than LD in distinguishing between intermediate-risk and high-risk GISTs or high-risk and other risk GISTs.

Acknowledgments We thank all the authors who contributed to the article.

Author contributions Wenwu Li performed the conception and design of this manuscript. Shengcai Wei performed the data collection and analysis. Xiaorong Sun, Liang Xu, Wanhu Li, and Yun $\mathrm{Li}$ performed the collection of imaging and revision of article. Shengcai Wei, Xiaorong Sun, and Shoufang Guo performed the manuscript writing.

Funding Information The authors state that this work has not received any funding.

\section{Compliance with ethical standards}

Guarantor The scientific guarantor of this publication is Wenwu Li.

Conflict of interest The authors of this manuscript declare no relationships with any companies, whose products or services may be related to the subject matter of the article. 
Statistics and biometry No complex statistical methods were necessary for this paper.

Informed consent Written informed consent was waived by the Institutional Review Board.

Ethical approval Institutional Review Board approval was obtained.

\author{
Methodology \\ - retrospective \\ - diagnostic or prognostic study \\ - performed at one institution
}

Open Access This article is distributed under the terms of the Creative Commons Attribution 4.0 International License (http:// creativecommons.org/licenses/by/4.0/), which permits unrestricted use, distribution, and reproduction in any medium, provided you give appropriate credit to the original author(s) and the source, provide a link to the Creative Commons license, and indicate if changes were made.

\section{References}

1. Tirumani SH, Baheti AD, Tirumani H, O'Neill A, Jagannathan JP (2017) Update on gastrointestinal stromal tumors for radiologists. Korean J Radiol 18:84-93

2. Joensuu H (2008) Risk stratification of patients diagnosed with gastrointestinal stromal tumor. Hum Pathol 39:1411-1419

3. Zhou C, Duan X, Zhang X, Hu H, Wang D, Shen J (2015) Predictive features of CT for risk stratifications in patients with primary gastrointestinal stromal tumor. Eur Radiol 26:3086-3093

4. Ghanem N, Altehoefer C, Furtwangler A et al (2003) Computed tomography in gastrointestinal stromal tumors. Eur Radiol 13: $1669-1678$

5. Iannicelli E, Carbonetti F, Federici GF et al (2017) Evaluation of the relationships between computed tomography features, pathological findings, and prognostic risk assessment in gastrointestinal stromal tumors. J Comput Assist Tomogr 41:271-278

6. Tateishi U, Hasegawa T, Satake M, Moriyama N (2003) Gastrointestinal stromal tumor correlation of computed tomography findings with tumor grade and mortality. J Comput Assist Tomogr 27:792-798

7. Czarnek N, Clark K, Peters KB, Mazurowski MA (2016) Algorithmic three-dimensional analysis of tumor shape in MRI improves prognosis of survival in glioblastoma: a multiinstitutional study. J Neurooncol 132:55-62

8. Okabe H, Hashimoto D, Chikamoto A et al (2016) Shape and enhancement characteristics of pancreatic neuroendocrine tumor on preoperative contrast-enhanced computed tomography may be prognostic indicators. Ann Surg Oncol 24:1399-1405

9. Benjamin RS, Choi H, Macapinlac HA et al (2007) We should desist using RECIST, at least in GIST. J Clin Oncol 25:1760-1764

10. Kalkmann J, Zeile M, Antoch G et al (2012) Consensus report on the radiological management of patients with gastrointestinal stromal tumors (GIST): recommendations of the German GIST Imaging Working Group. Cancer Imaging 12:126-135

11. Kim JS, Kim HJ, Park SH, Lee JS, Kim AY, Ha HK (2016) Computed tomography features and predictive findings of ruptured gastrointestinal stromal tumors. Eur Radiol 27:2583-2590
12. Rimondini A, Belgrano M, Favretto G et al (2007) Contribution of CT to treatment planning in patients with GIST. Radiol Med 112: 691-702

13. Ulusan S, Koc Z, Kayaselcuk F (2008) Gastrointestinal stromal tumors: CT findings. Br J Radiol 81:618-623

14. Folio LR, Sandouk A, Huang J, Solomon JM, Apolo AB (2013) Consistency and efficiency of CT analysis of metastatic disease: semiautomated lesion management application within a PACS. AJR Am J Roentgenol 201:618-625

15. Monsky WL, Raptopoulos V, Keogan MT et al (2004) Reproducibility of linear tumor measurements using PACS: comparison of caliper method with edge-tracing method. Eur Radiol 14:519-525

16. Alilou M, Beig N, Orooji M et al (2017) An integrated segmentation and shape based classification scheme for distinguishing adenocarcinomas from granulomas on lung CT. Med Phys 44:35563569

17. Zanoni M, Piccinini F, Arienti C et al (2016) 3D tumor spheroid models for in vitro therapeutic screening: a systematic approach to enhance the biological relevance of data obtained. Sci Rep 6:19103

18. Cheng G, Tse J, Jain RK, Munn LL (2009) Micro-environmental mechanical stress controls tumor spheroid size and morphology by suppressing proliferation and inducing apoptosis in cancer cells. PLoS One 4:e4632

19. Mazurowski MA, Clark K, Czarnek NM, Shamsesfandabadi P, Peters KB, Saha A (2016) Radiogenomics of lower-grade glioma: algorithmically-assessed tumor shape is associated with tumor genomic subtypes and patient outcomes in a multi-institutional study with The Cancer Genome Atlas data. J Neurooncol 133:27-35

20. Dematteo RP, Gold JS, Saran L et al (2008) Tumor mitotic rate, size, and location independently predict recurrence after resection of primary gastrointestinal stromal tumor (GIST). Cancer 112:608615

21. Liu X, Qiu H, Zhang P et al (2018) Prognostic factors of primary gastrointestinal stromal tumors: a cohort study based on highvolume centers. Chin J Cancer Res 30:61-71

22. Menon SS, Guruvayoorappan C, Sakthivel KM, Rasmi RR (2019) Ki-67 protein as a tumor proliferation marker. Clin Chim Acta 491: $39-45$

23. Li H, Ren G, Cai R, Chen J, Wu X, Zhao J (2018) A correlation research of Ki67 index, CT features, and risk stratification in gastrointestinal stromal tumor. Cancer Med 7:4467-4474

24. Kemmerling R, Weyland D, Kiesslich T et al (2014) Robust linear regression model of $\mathrm{Ki}-67$ for mitotic rate in gastrointestinal stromal tumors. Oncol Lett 7:745-749

25. Kim H-C, Lee JM, Kim KW et al (2004) Gastrointestinal stromal tumors of the stomach: $\mathrm{Ct}$ findings and prediction of malignancy. AJR Am J Roentgenol 183:893-898

26. Chen T, Xu L, Dong X et al (2019) The roles of CT and EUS in the preoperative evaluation of gastric gastrointestinal stromal tumors larger than $2 \mathrm{~cm}$. Eur Radiol 29:2481-2489

27. Liu S, Pan X, Liu R et al (2018) Texture analysis of CT images in predicting malignancy risk of gastrointestinal stromal tumors. Clin Radiol 73:266-274

28. Choi H, Charnsangavej C, de Castro Faria S et al (2004) CT evaluation of the response of gastrointestinal stromal tumors after imatinib mesylate treatment: a quantitative analysis correlated with FDG PET findings. AJR Am J Roentgenol 183:1619-1628

Publisher's note Springer Nature remains neutral with regard to jurisdictional claims in published maps and institutional affiliations. 